\title{
Diagnosis of cytomegalovirus infection in heart transplant recipients
}

\author{
P S Rice, G Kudesia, C Price, G H Smith
}

\begin{abstract}
In a prospective study eight (47\%) heart transplant recipients had recurrent cytomegalovirus (CMV) infection. Of these, five had received hearts from seropositive donors. Neither serology nor virus isolation from urine was reliably associated with clinical CMV infection, but a high CMV IgM index correlated with CMV infection.
\end{abstract}

Cytomegalovirus (CMV) infection remains one of the leading causes of morbidity and mortality in heart transplant recipients. ${ }^{1}$ Primary infections are mostly preventable by donor and recipient screening and by using only screened blood for transfusion. However, with intensive immunosuppressive regimens, CMV often becomes reactivated in the first few months after transplantation in seropositive patients. Reinfections with donor strains also occur. Such recurrent infections are rarely fatal, but considerable morbidity is seen in these patients. As effective treatment for CMV has become available with the introduction of gancyclovir, rapid diagnosis of CMV disease becomes critically important to patient management.

\section{Methods}

Between April 1990 and May 1991, 19 cardiac transplantations were performed at this hospital. All patients received the same immunosuppressive regimen of cyclosporin A, azathioprine, and prednisolone. The CMV antibody status of both donors and recipients was determined before surgery. After transplantation, blood and urine samples were obtained weekly for eight weeks and monthly thereafter, with additional samples being submitted on clinical indication. Detection of antibodies to CMV was performed using the complement fixation test (CFT), with antigen supplied by the Public Health Laboratory Service (PHLS), by latex agglutination (CMV-Scan; BectonDickinson), and by an enzyme immunoassay (EIA; Abbott Diagnostics IMX). Urine samples were processed using the technique of detection of early antigen fluorescent foci (DEAFF). ${ }^{2}$

Rises in the CFT or latex agglutination titre of at least four-fold and IgM values above the cutoff of 0.8 in the EIA were taken to be significant. Slides in the DEAFF test were examined by two observers.

\section{Results}

Of the 17 surviving patients, eight (47\%) experienced recurrent $\mathrm{CMV}$ infection; there was no evidence of CMV infection in the remaining nine patients. There were no primary infections.

Of the eight patients with recurrent infection, five $(63 \%)$ had received hearts from CMV seropositive donors, the remaining three receiving hearts from seronegative donors. Of the nine patients without evidence of CMV infection, seven had been CMV antibody positive before transplantation and three (43\%) had received a heart from seropositive donors. The figure shows a summary of the relevant laboratory findings in the eight cases with recurrent CMV infection.

All cases showed at least four-fold rises in CMV antibody titre by CFT. Latex agglutination failed to detect a rise in titre in only one patient; but virus specific IgM was present in seven of eight patients. CMV was detected by DEAFF in the urine of six of eight patients (figure). It is also clear that no single test could be relied on to indicate recurrent CMV infection in our group of patients. We did, however, find that CMV specific IgM indices (reference OD/cutoff OD) could be correlated with the clinical condition of the patient. Four patients with clinical evidence of CMV infection-that is swinging fever together with either pancytopenia or pneumonia, all had IgM indices of $>2 \cdot 4$. By comparison, in the four patients without clinical CMV disease the CMV IgM

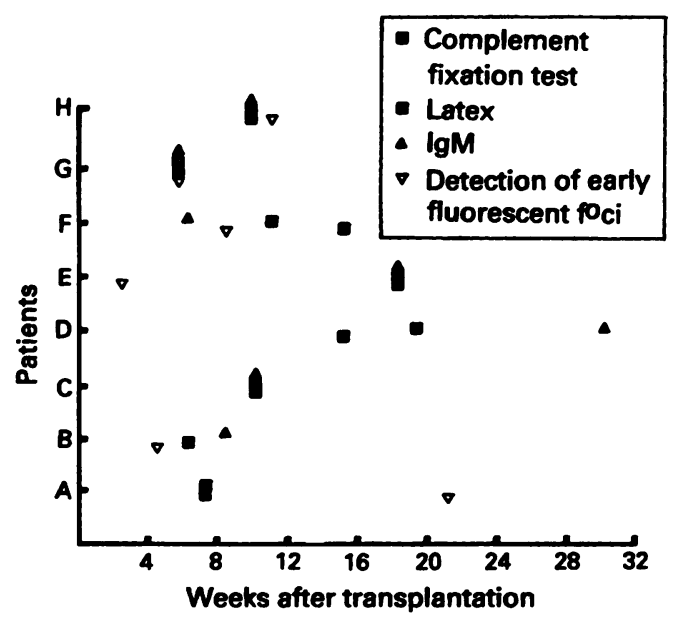

The first clinically important serological and isolation results from eight patients with recurrent $C M V$ infection. \\ Public Health \\ P S Rice \\ Cardio-Thoracic \\ Surgery, Northern \\ Sheffield \\ G H Smith
}


index did not rise above $1 \cdot 1$. Interestingly, in the patient with no detectable IgM and in whom a swinging fever was the only evidence of CMV infection, no response to gancyclovir was observed.

\section{Discussion}

This series is small but it does highlight some of the current ideas on CMV infection and its diagnosis in heart transplant recipients.

The first point to note is that, thus far, there have been no primary infections. This is undoubtedly because only two CMV seronegative patients have received transplantations to date, both donors also being CMV negative. This re-emphasises the point made by others ${ }^{3}$ that it is important to determine donor and recipient $C M V$ antibody status before transplantation and to use only blood that has been screened for CMV. This may, however, present practical problems as otherwise suitable organs may have to be rejected. In case of a CMV positive organ being transplanted into a CMV negative recipient, prophylaxis with hyperimmune CMV globulin is recommended. ${ }^{4}$

Reactivation and reinfection with CMV continue to be a major problem, with eight out of $17(47 \%)$ of the patients experiencing such episodes. This compares with the much larger Papworth series in which a reactivation or reinfection rate of $31 \cdot 1 \%$ was found. ${ }^{5}$ In addition, one of the risk factors for developing such disease is the CMV antibody status of the donor. Thus, of those who developed recurrent infection, $63 \%$ had received a heart from a seropositve donor compared with $37 \%$ receiving a heart from a seronegative one. The numbers in our study are too small for analysis, but they are in line with the Papworth experience in which their respective percentages were $62 \%$ and $42 \%,{ }^{4}$ indicating a higher risk of CMV infection if the organ is from a seropositive donor. The correlation of $\mathrm{CMV}$ infection and CMV disease continues to present difficulties. We found that in six of the eight affected patients, at least two markers of CMV infection had become positive by 10 weeks, but no reliable pattern was observed. This, of course, may have been due to the small numbers involved, but a recent study on renal transplant recipients ${ }^{6}$ also concluded that serology was of limited value in the diagnosis of CMV reinfection or reactivation.

We noted, however, that those patients with recurrent infection who had clinical CMV disease and required treatment with gancyclovir all had IgM indices of $>2 \cdot 4$. The remaining patients, of whom one was subsequently treated with gancylovir for a spiking fever, without any benefit, had IgM indices ranging from undetectable to $1 \cdot 1$. Although these results will have to be confirmed with larger numbers of patients, they have certain implications. Furthermore, Chan et al have shown that in renal transplant recipients higher concentrations of IgM are produced in patients reinfected with the donor strain of $\mathrm{CMV}^{7}$ It may be, then, that IgM is produced in greater quantities in those transplant recipients who are reinfected with the new donor strain. Because IgM is primarily an intravascular immunoglobulin, these patients may have viraemia during their illness, and detection of viraemia may become the "gold standard" of virological investigation. Therefore, modification of the DEAFF technique to detect viraemia may provide an investigation that is both rapid and of good predictive value in the diagnosis of CMV infection.

We thank Duncan Whittaker for technical assistance with DEAFF and the staff of the serology laboratory at PHL, Sheffield.

1 Wreghitt TG, Hakim M, Cory-Pearce R, English TAH, Wallwork J. The impact of donor transmitted CMV and Toxoplasma gondii disease in cardiac transplantation. Transplant Proc 1986;XVIII:1375-6.

2 Griffiths PD, Panjwani DD, Stirk PK, et al. Rapid diagnosis of cytomegalovirus infection in immunocompromised patients by detection of early antigen fluorescent foci. Lancet 1984;ii:1242-5.

3 Wreghitt T, Hakim M, Gray J, Kucia S, Wallwork J, English TAH. Cytomegalovirus infection in heart and heart and lung trantant recipients. F Clin Pathol 1988;41:660-7.

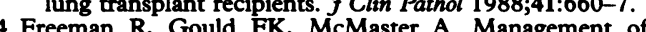
cytomegalovirus antibody negative patients undergoing cytomegalovirus antibody negative patients undergo

5 Wreghitt T. Cytomegalovirus infections in heart and heartlung tranplant recipients. $\mathcal{f}$ Antimicrob Chemother $1989 ; 23: 49-60$

6 Dolan J, Briggs JD, Clements GB. Antibodies to cytomegalovirus in renal allograft recipients: correlation with isolation of virus. $\mathcal{J}$ Clin Pathol 1989;42:1070-7.

7 Chan S, Kim DY, Scott KM, Saval DL. Immunoglobulin M to cytomegalovirus in primary and reactivation infection in renal transplant patients. $f$ Clin Microbiol 1987;25:52-5.

8 Gerna G, Zipeto D, Parea M, et al. Monitoring of human cytomegalovirus infections and ganciclovir treatment in heart transplant recipients by determination of viremia, antigemia and DNAemia. $\mathcal{F}$ Infect Dis 1991;164:488-98. 